\title{
Nucleoprotein Complexes of Minute Virus of Mice Have a Distinct Structure Different from That of Chromatin
}

\author{
C. DOERIG,${ }^{1}$ G. McMASTER,${ }^{1}$ J. SOGO,${ }^{2}$ H. BRUGGMANN,${ }^{1}$ AND P. BEARD ${ }^{1 *}$ \\ Swiss Institute for Experimental Cancer Research, 1066 Epalinges,$^{1}$ and Institute of Cell Biology, Eidgenossische \\ Technische Hochschule, 8093 Zürich, ${ }^{2}$ Switzerland
}

Received 30 September 1985/Accepted 18 February 1986

\begin{abstract}
We studied the structure of viral nucleoprotein complexes extracted from the nuclei of mouse cells infected with the immunosuppressive strain of the minute virus of mice (MVMi). Two types of complex were detected, with sedimentation coefficients of about 110 and $40 \mathrm{~S}$. The complexes sedimenting at $110 \mathrm{~S}$ contained single-stranded MVMi DNA as well as a second form of viral DNA which apparently had a heat-sensitive secondary structure. The $110 \mathrm{~S}$ peak also contained proteins which coelectrophoresed with the MVMi capsid proteins. Complexes sedimenting at $40 S$ contained the double-stranded replicative form of MVMi DNA. These complexes sedimented faster than did the pure replicative form DNA (15S), but more slowly than cellular chromatin fragments containing DNA of the same length. They incorporated labeled deoxynucleoside triphosphate in vitro into the replicative form DNA. We investigated the structure of MVMi nucleoprotein complexes in the following ways. Nuclei of MVMi-infected cells were digested with staphylococcal nuclease, and the resulting DNA fragments were electrophoresed, transferred to nitrocellulose, and hybridized first with labeled MVMi DNA and then with cellular DNA. A nucleosomal repeat pattern was seen with the cellular DNA probe but not with the MVMi DNA probe. The DNA in MVMi nucleoprotein complexes was cross-linked with psoralen, purified, denatured, and examined with an electron microscope. Bubbles, indicating the presence of proteins, were seen in the MVMi DNA. The length of the DNA in the bubbles was $90 \pm 29$ nucleotides. On the other hand, nucleosomes protected 160 base pairs from cross-linking by psoralen. The MVMi nucleoprotein complexes thus have a distinct structure which is different from that of chromatin.
\end{abstract}

The minute virus of mice (MVM) is a member of the autonomous parvovirus group (reviewed by Ward and Tattersall [35] and Berns [5]). The small icosahedral MVM virions contain a linear DNA molecule about 5.1 kilobases long which is mostly single-stranded but with base-paired hairpins at each end $(5,35)$. In the infected cell, the virion DNA is converted to a double-stranded replicative form (RF). The MVM genome encodes the three proteins which make up the viral capsid, VP-1, VP-2, and VP-3, as well as two nonstructural proteins, NS-1 and NS-2 (10). The functions of NS-1 and NS-2 are not yet clear.

An immunosuppressive variant of MVM was reported by Bonnard et al. (6), and we showed that the variant, MVMi, is similar in structure and closely related in DNA restriction pattern to the prototype strain MVMp (22). Nevertheless, MVMi and MVMp have different biological properties. MVMi grows in cells derived from lymphoid tissue, and it inhibits various functions mediated by murine $\mathrm{T}$ lymphocytes in vitro (12). On the other hand, MVMp grows in fibroblasts and has not been found to inhibit T-lymphocyte functions. The nucleotide sequence of the genome of MVMp is known (1). The sequence of the genome of MVMi has recently been determined (26), and comparison of the two viral genomes shows regions of sequence variation that probably determine their distinct specificities for the differentiated state of the host cells.

To understand the interactions between MVMi and MVMp and their host cells, we need to know which proteins associate with the viral DNAs. In the work reported here, we have studied the nucleoprotein structures of MVMi extracted from infected cells. In particular we were inter-

\footnotetext{
* Corresponding author.
}

ested in whether intracellular MVMi DNA is organized into structures similar to or different from chromatin.

\section{MATERIALS AND METHODS}

MVMi, MVMp, and their host cells, the EL-4 lymphoma line and A9 fibroblasts, were described by McMaster et al. (22). The cells were grown as monolayers in Dulbecco modified Eagle medium containing $5 \%$ calf serum. For infection, the medium was removed from cultures that were between 30 and $50 \%$ confluent and replaced by $0.4 \mathrm{ml}$ of virus suspension (generally an infected cell lysate) per $9-\mathrm{cm}$ plate. After 90 min at $37^{\circ} \mathrm{C}, 10 \mathrm{ml}$ of medium with $2 \%$ calf serum was added, and the infection continued for $24 \mathrm{~h}$. At times noted, at $24 \mathrm{~h}$ after infection cells were labeled with $\left[{ }^{3} \mathrm{H}\right] l y$ sine $(75$ $\mathrm{Ci} / \mathrm{mmol}, 100 \mu \mathrm{Ci} / \mathrm{ml}$ ) in Earle saline for $30 \mathrm{~min}$, followed by a chase for 30 min with medium containing $2 \%$ calf serum.

Except where noted otherwise, the experiments were done with MVMi. To isolate MVMi nucleoprotein complexes, infected EL-4 cells were washed with cold phosphate-buffered saline, then harvested gently into HBE (10 mM HEPES [ $N$-2-hydroxyethylpiperazine- $N$ '-2-ethanesulfonic acid]-KOH [pH 7.8], $5 \mathrm{mM} \mathrm{KCl,} 1 \mathrm{mM}$ EDTA). EL-4 cells attached only loosely to the plastic surface. The cells were pelleted for $3 \mathrm{~min}$ at $1,000 \times g$ in the Sorvall HB4 rotor (Ivan Sorvall, Inc., Norwalk, Conn.). The pellet was suspended in HBE, and the cells were lysed by dropwise addition, with mixing, of $1 \%$ Nonidet P-40 to give a final concentration of $0.1 \%$. This suspension was centrifuged for $2 \mathrm{~min}$ at $1,000 \times g$ to give a loose pellet of swollen or lysed nuclei. To this material in $\mathrm{HBE}$ was added $\mathrm{NaCl}$ to a final concentration of $0.2 \mathrm{M}$, and the suspension was left for 1 to $4 \mathrm{~h}$ on ice. The precipitated cellular chromatin was pelleted at $10,000 \times g$ for $10 \mathrm{~min}$, and the supernatant containing the 
MVMi nucleoprotein complexes was stored on ice. For sucrose gradient velocity centrifugation, this crude extract was layered onto a 5 to $20 \%$ (wt/vol) gradient of sucrose in $\mathrm{HBE}$ containing $0.2 \mathrm{M} \mathrm{NaCl}$. Centrifugation was at 164,000 $\times g$ for 65 min at $4^{\circ} \mathrm{C}$ in the Beckman SW60 rotor (Beckman Instruments, Inc., Fullerton, Calif.). After centrifugation, about 20 fractions were collected dropwise from the bottom of the tube. MVMp nucleoprotein complexes were extracted 20 to $24 \mathrm{~h}$ after infection of A9 cells by the method previously used for simian virus 40 (SV40) (2).

Gel electrophoresis of DNA through 1\% agarose (type II; Sigma Chemical Co., St. Louis, Mo.) was either in $40 \mathrm{mM}$ Tris hydrochloride [ $\mathrm{pH} 7.8]-10 \mathrm{mM}$ EDTA-5 mM sodium acetate, by using a horizontal electrophoresis apparatus 20 $\mathrm{cm}$ long, or in $89 \mathrm{mM}$ Tris-89 $\mathrm{mM}$ boric acid-2 mM EDTA, by using a minigel apparatus $7.5 \mathrm{~cm}$ long. Samples were prepared in $10 \%$ glycerol- $1 \%$ sodium dodecyl sulfate$0.02 \%$ bromophenol blue; where noted they were digested with pronase, 100 to $500 \mu \mathrm{g} / \mathrm{ml}$, at $37^{\circ} \mathrm{C}$ for $15 \mathrm{~min}$ before electrophoresis. After staining in ethidium bromide (1 $\mu \mathrm{g} / \mathrm{ml}$ ), gels were photographed with Polaroid 667 film under UV transillumination.

Proteins present in gradient fractions were analyzed by electrophoresis in $15 \%$ polyacrylamide gels (17). Proteins were either stained with silver (24) or detected by fluorography (18).

DNA polymerase activity in sucrose gradient fractions was assayed by a modification of the method of Hübscher (14). The fractions to be tested were diluted threefold into a reaction mix, giving final concentrations of $10 \mathrm{mM}$ HEPES$\mathrm{KOH}$ [pH 7.8], $2 \mathrm{mM} \mathrm{KCl}, 65 \mathrm{mM} \mathrm{NaCl}, 0.3 \mathrm{mM}$ EDTA, 100 $\mu \mathrm{g}$ of bovine serum albumin per $\mathrm{ml}, 4 \mathrm{mM}$ dithiothreitol, 10 $\mathrm{mM} \mathrm{MgCl} 2,50 \mu \mathrm{M}$ each dGTP, dTTP, and dCTP, and $25 \mu \mathrm{M}$ $\left[{ }^{32} \mathrm{P}\right] \mathrm{dATP}(5 \mu \mathrm{Ci} / 100-\mu$ l reaction). Reactions were stopped after $30 \mathrm{~min}$ at $37^{\circ} \mathrm{C}$ by the addition of sodium dodecyl sulfate to a $1 \%$ concentration, and the unincorporated radioactivity was removed on a small column of Bio-Gel A 15-M (Bio-Rad Laboratories, Richmond, Calif.). Material in the excluded volume was precipitated with ethanol and analyzed by gel electrophoresis and autoradiography.

To prepare nuclei for staphylococcal nuclease digestion, cells were swollen in lysis buffer containing $15 \mathrm{mM}$ Tris hydrochloride [pH 7.9], $25 \mathrm{mM} \mathrm{KCl}$, and $2 \mathrm{mM} \mathrm{MgCl}_{2}$, then lysed with a Dounce homogenizer. Nuclei were pelleted at $1,000 \times g$ for $2 \mathrm{~min}$ in the HB4 rotor, washed once in lysis buffer containing $0.34 \mathrm{M}$ sucrose, and suspended again in $\mathbf{0 . 2}$ $\mathrm{ml}$ of the same buffer per original plate of cells. $\mathrm{CaCl}_{2}$ was added to a concentration of $2 \mathrm{mM}$, followed by $100 \mathrm{U}$ of staphylococcal nuclease per $\mathrm{ml}$ (Worthington Diagnostics, Freehold, N.J.). Samples were removed at time zero and after incubation for $1,2,5$, and $10 \mathrm{~min}$ at $37^{\circ} \mathrm{C}$. They were adjusted to $10 \mathrm{mM}$ ethylene glycol-bis( $\beta$-aminoethyl ether)$N, N, N^{\prime}, N^{\prime}$-tetraacetic acid (EGTA) and $60 \mu \mathrm{g}$ of RNase A per $\mathrm{ml}$ and were incubated at $37^{\circ} \mathrm{C}$ for $10 \mathrm{~min}$. Sodium dodecyl sulfate $(0.5 \%)$ and pronase $(100 \mu \mathrm{g} / \mathrm{ml})$ were then added, and the incubation continued for $15 \mathrm{~min}$. The DNA was purified by extraction with phenol-chloroform, precipitated with ethanol, and electrophoresed in a gel of $1.5 \%$ agarose in the Tris-acetate buffer. DNA was transferred to a nitrocellulose filter (28) and hybridized with two radioactive DNA probes in succession. The first probe was RF DNA cloned in pBR322 (26). After hybridization, washing, and exposure for autoradiography (20), the filter was rinsed in 10 $\mathrm{mM}$ Trishydrochloride-1 $\mathrm{mM}$ EDTA [pH 7.9] at $95^{\circ} \mathrm{C}$ to remove the hybridized DNA probe. The second probe was mouse DNA purified from uninfected EL-4 cells. Both probes were labeled to $10^{7} \mathrm{cpm} / \mu \mathrm{g}$ by nick translation (25). After hybridization and washing, the filter was reexposed for autoradiography.

For psoralen cross-linking, $450 \mu \mathrm{l}$ of nuclear extract containing MVMi nucleoprotein complexes was placed in the bottom of a small plastic petri dish. 4,5',8-Trimethylpsoralen (Paul B. Elder Co.) in ethanol was added to give a final concentration of $10 \mu \mathrm{g} / \mathrm{ml}$, and the solution was left to stand for $10 \mathrm{~min}$ on ice. The solution on ice was irradiated with 366-nm UV light (Ultra-Violet Products Inc., San Gabriel, Calif.) for $4 \mathrm{~h}$; more psoralen was added to make a final concentration of $10 \mu \mathrm{g} / \mathrm{ml}$ after about 1 and $2 \mathrm{~h}$ of irradiation. The solution was adjusted to $10 \mu \mathrm{g}$ of RNase per $\mathrm{ml}$ and incubated at $37^{\circ} \mathrm{C}$ for $15 \mathrm{~min}$, then sodium dodecyl sulfate was added to $1 \%$ (wt/vol) and proteinase $\mathrm{K}$ was added to a concentration of $200 \mu \mathrm{g} / \mathrm{ml}$, and the incubation was continued for $1 \mathrm{~h}$. DNA was purified by extraction with phenol and precipitation with ethanol, then prepared for electron microscopy as described by Sogo et al. (27). SV40 chromosomes extracted from infected TC7 cells as described by $\mathrm{Su}$ and DePamphilis (29) were cross-linked, and the DNA was prepared for electron microscopy by the same procedure (J. M. Sogo, H. Stahl, T. Koller, and R. Knippers, J. Mol. Biol., in press).

\section{RESULTS}

Extraction of MVMi nucleoprotein complexes. MVMi nucleoprotein complexes were extracted from the nuclei of EL-4 cells $24 \mathrm{~h}$ after viral infection. When the nucleic acids in the crude extract were analyzed by agarose gel electrophoresis, two major virus-specific DNA bands were detected (Fig. 1). One of these comigrated with a marker of singlestranded MVMi virion DNA. The migration rate of the other corresponded to that of a double-stranded DNA of 5.1 kilobases, characteristic of MVM RF DNA. Neither band was present in extracts of uninfected cells (data not shown). The extracts also contained variable amounts of an MVM-

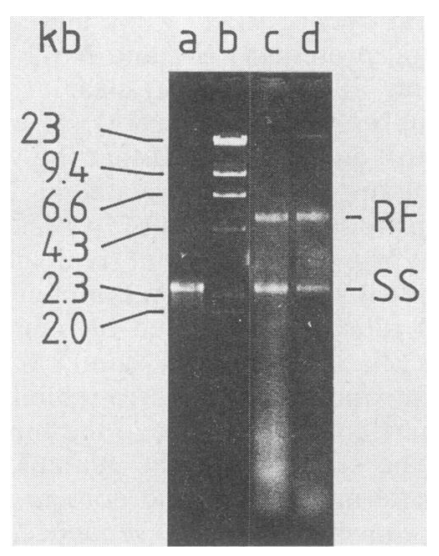

FIG. 1. DNA present in a crude extract of MVMi nucleoprotein complexes. (a) Single-stranded DNA from MVMi virus particles. (b) Molecular weight markers from phage $\lambda$ DNA digested with HindIII; their lengths in kilobases are shown on the left. (c) Nuclear extract containing MVMi nucleoprotein complexes. (d) Sample as in lane c but treated with RNase $(5 \mu \mathrm{g} / \mathrm{ml})$ for $15 \mathrm{~min}$ at $37^{\circ} \mathrm{C}$. The positions of single-stranded (SS) and replicative form (RF, $5.1 \mathrm{~kb})$ DNAs are shown. MVMi-containing samples were treated with $1 \%$ sodium dodecyl sulfate and pronase $(500 \mu \mathrm{g} / \mathrm{ml})$ for $15 \mathrm{~min}$ at $37^{\circ} \mathrm{C}$ before addition of bromophenol blue-glycerol and loading on the gel. After electrophoresis, the gel was stained with ethidium bromide. 
specific DNA which, after deproteinizing, migrated between the positions of the single- and double-stranded DNAs (its properties will be discussed below), ribosomal subunits, and fragments of cellular chromatin (Fig. 2; see Fig. 5).

An extract of MVMi-infected cells similar to that shown in Fig. 1 was analyzed by velocity sedimentation through a sucrose gradient. Nucleic acids in the gradient fractions were detected by agarose gel electrophoresis of samples after the addition of sodium dodecyl sulfate (Fig. 2). The extract shown contained the 40 and $60 \mathrm{~S}$ ribosomal subunits which served as sedimentation markers in the gradient. Two peaks of virus-specific DNA were seen, with sedimentation coefficients of approximately $110 \mathrm{~S}$ (extrapolated from the markers) and about $40 \mathrm{~S}$. The sedimentation coefficient of MVM virions is known to be $110 \mathrm{~S}$ (35).

The DNA in the $110 \mathrm{~S}$ peak showed a predominant band and a minor band after electrophoresis. The predominant band migrated to the same position as did DNA purified from MVMi virions. The other band migrated more slowly between the positions of virion DNA and RF DNA (see Fig. 5 for another example). We called this DNA “band X'. It was MVM-specific, as shown by transfer to a nitrocellulose filter and hybridization with a probe of MVM DNA. Its electrophoretic migration was unaffected by prior digestion with pronase; however, after treatment either with $0.3 \mathrm{M} \mathrm{NaOH}$ or at $100^{\circ} \mathrm{C}$ for $5 \mathrm{~min}$, all the DNA from $110 \mathrm{~S}$ peak migrated to the position of virion DNA (data not shown). Band $X$ therefore seemed to be a form of MVMi DNA having a particular secondary structure distinct from both the virion and replicative forms. After sodium dodecyl sulfate was added, the $40 \mathrm{~S}$ peak yielded DNA which migrated close to the position of MVMi RF but did not yeld any detectable virion DNA.

The proteins in the extract used for the experiment shown in Fig. 2 had been labeled with $\left[{ }^{3} \mathrm{H}\right]$ lysine during the viral

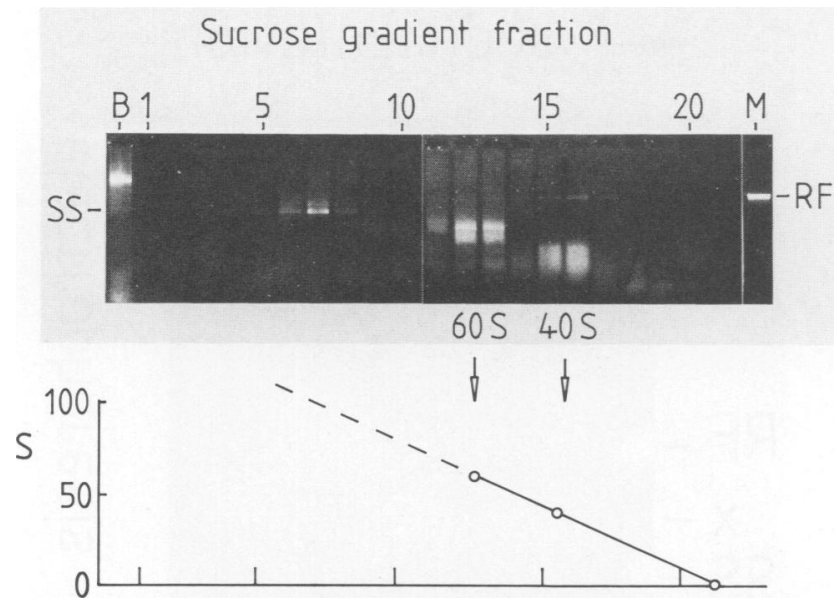

FIG. 2. Sucrose gradient velocity centrifugation of a nuclear extract containing MVMi nucleoprotein complexes and ribosomal subunits. Sedimentation was from right to left. Top, Samples from each fraction were adjusted to a $1 \%$ concentration of sodium dodecyl sulfate and analyzed by gel electrophoresis and ethidium bromide staining. M, Marker of MVMi RF DNA; B, material recovered from the bottom of the centrifuge tube. The positions on electrophoresis of single-stranded (SS) and replicative form (RF) MVMi DNAs are shown, as are the positions of the 60 and $40 S$ ribosomal subunits (rRNA). Bottom, Sedimentation coefficients (S) of the ribosomal subunits plotted against gradient fraction number. The line was extrapolated to give an estimate of the sedimentation coefficient of the faster-sedimenting MVMi complex. infection. Proteins present in the gradient fractions were analyzed by polyacrylamide gel electrophoresis (Fig. 3A and B) alongside molecular weight markers, MVMi virions, and histone markers. Two separate gels were used. In one, the proteins were detected by fluorography, and in the other, they were detected by silver staining. The $\left[{ }^{3} \mathrm{H}\right] l y$ sine labeling was done to facilitate the detection of basic proteins, but in fact the two methods yielded similar results. The most prominent proteins cosedimenting with the $110 \mathrm{~S}$ peak (fraction 7) were the MVM capsid proteins VP-1, VP-2, and VP-3. Several other proteins were present in lesser amounts, but histones were not visible. This experiment did not allow us to identify which proteins were associated with viral DNA in the $40 \mathrm{~S}$ peak because many cellular components, including ribosomal subunits, sedimented to this position, and in addition, the gel was heavily loaded to allow the detection of faint bands. A slightly different method was used to extract MVMp nucleoprotein complexes from A9 cells (see the Materials and Methods section), which generally gave less contamination by ribosomal subunits. With this material, capsid proteins could readily be detected in both the 110 and the $40 \mathrm{~S}$ regions (Fig. $3 \mathrm{C}$ ).

Fractions of a sucrose gradient similar to that shown in Fig. 2 were incubated with radioactive deoxyribonucleoside triphosphates under conditions allowing DNA synthesis by endogenous DNA polymerase (12). DNA was isolated from the reactions and electrophoresed with molecular weight markers on an agarose gel which was stained with ethidium bromide, photographed, then dried and autoradiographed (Fig. 4). A radioactive band close to the position of RF DNA was seen with the sample corresponding to the $40 \mathrm{~S}$ peak from the sucrose gradient. The $110 \mathrm{~S}$-peak fraction showed no incorporation of radioactive precursor. This suggests that the MVMi nucleoprotein complexes sedimenting at $40 \mathrm{~S}$ included complexes engaged in DNA replication.

MVMi nucleoprotein complexes compared with cellular chromatin. The sedimentation rate of MVMi nucleoprotein complexes was compared with the sedimentation rate of fragments of cellular chromatin. Nuclear extracts containing MVMi nucleoprotein complexes occasionally contained, in addition, significant amounts of fragmented cellular chromatin. Such an extract was sedimented through a sucrose gradient, and the DNA in the gradient fractions was analyzed by electrophoresis in an agarose gel alongside markers of single- and double-stranded MVMi DNA (Fig. 5). The MVMi nucleoprotein complexes sedimented as before, in two peaks containing the virion form of DNA, with the band $X$ (fraction 5) and RF DNA (fraction 15), respectively. The DNA from cellular chromatin fragments, separated first according to sedimentation rate, then by DNA length on electrophoresis, formed a smooth curve roughly diagonal on the gel from upper left to lower right. It was apparent that MVMi nucleoprotein complexes containing RF DNA sedimented more slowly than cellular chromatin fragments containing DNA of the same length (fraction 9).

The structures of MVMi nucleoprotein complexes and cellular chromatin were also compared by digestion with staphylococcal nuclease. Nuclei were isolated from EL-4 cells infected with MVMi and from uninfected control cells. They were digested with staphylococcal nuclease for various times, and the DNA was purified and analyzed by gel electrophoresis. On the ethidium-stained gel (Fig. 6a), the typical series of bands which result from the repeating nucleosomal structure of chromatin (13) were seen from both infected and uninfected cells. The DNA in the gel was then denatured, transferred to a nitrocellulose filter, hybridized 


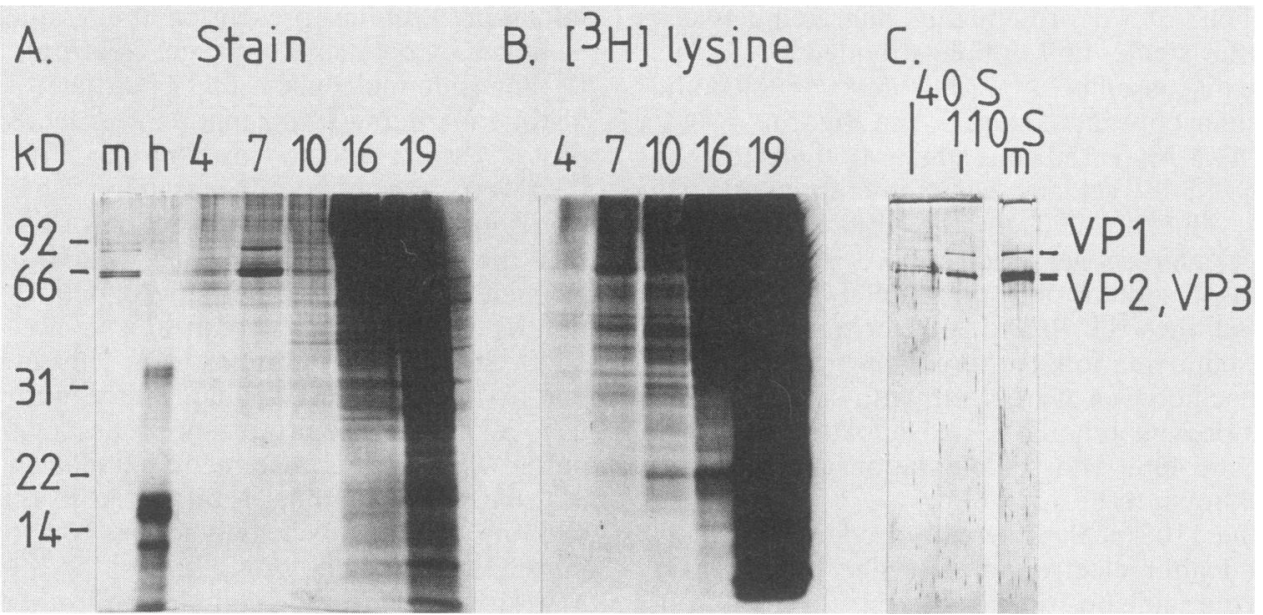

FIG. 3. Proteins cosedimenting with MVMi nucleoprotein complexes. Fractions of the sucrose gradient in Fig. 2 were analyzed for proteins by $15 \%$ polyacrylamide gel electrophoresis. The gels were either silver-stained (A) or fluorographed (B). Numbers over the gel tracks refer to the sucrose gradient fractions. Markers used were MVMi virus particles (m) and histones from calf thymus (h). The positions of the molecular weight markers are shown on the left. (C) MVMp nucleoprotein complexes were extracted from infected A9 cells as described in the Materials and Methods section and were analyzed by sucrose gradient centrifugation. Samples from the peak fractions containing viral DNA (110S) and RF DNA (40S) were electrophoresed as in panel A, and the proteins were visualized by silver staining. The marker (m) was MVMp virus particles.

with radioactively labeled cloned MVMi DNA, and autoradiographed (Fig. 6b). As expected, the DNA from uninfected cells showed no hybridization. (The faint signal at the position of MVM DNA in the third track of uninfected cell DNA hybridized with the MVM probe is believed to be due to contamination with viral material on loading the gel. No such cross-reaction was seen in other hybridizations.) The DNA from MVMi-infected cells showed, before nuclease digestion, two strongly hybridizing bands representing the replicative and single-stranded forms of MVMi DNA. With nuclease digestion, the upper of the two bands (RF DNA) disappeared, whereas the lower band (single-stranded virion DNA) was more resistant, some remaining even after the longest digestion time used.

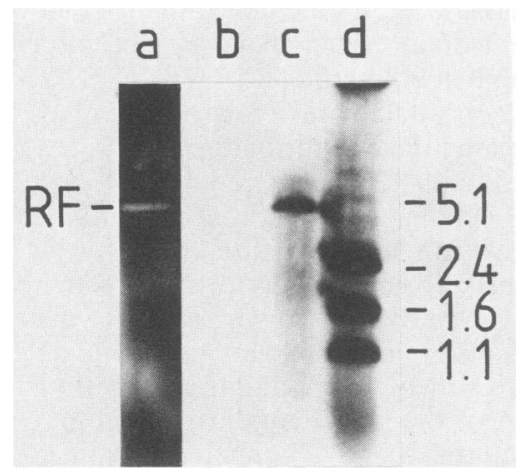

FIG. 4. DNA polymerase activity in sucrose gradient fractions containing MVMi nucleoprotein complexes. The 110 and 40S peak fractions from a sucrose gradient were tested for endogenous DNA polymerase activity (see the Materials and Methods section). After the reaction, DNA was isolated and analyzed by agarose gel electrophoresis. The gel was stained with ethidium bromide, photographed, then dried and autoradiographed. (a) MVMi RF DNA stained with ethidium bromide. (b) 110S peak fraction. (c) 40S peak fraction. (d) Molecular weight markers were from ${ }^{32} \mathrm{P}$-labeled RF DNA digested with EcoRI; DNA lengths in kilobases are given on the right.
With the disappearance of the band of RF DNA, a smear of hybridizable DNA was formed. This digestion pattern differed from the normal nucleosomal digestion pattern in two respects. First, although the smear was not uniform (several broad bands could be discerned within it), bands were absent from the positions corresponding to the nucleosomal oligomers. Second, there was little or no accumulation, with increasing nuclease digestion, of DNA of the

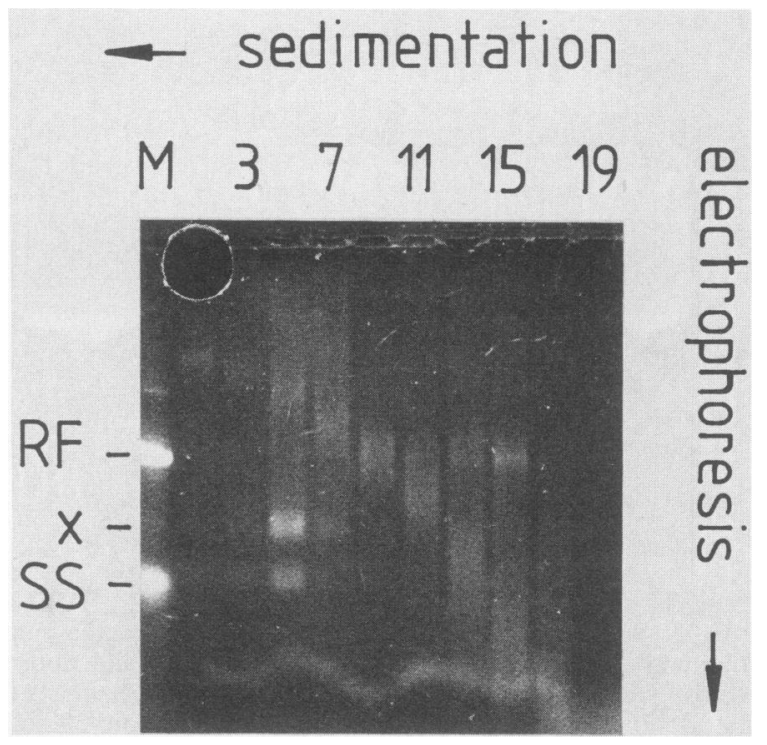

FIG. 5. Sucrose gradient velocity centrifugation of an extract containing MVMi nucleoprotein complexes and fragmented cellular chromatin. After centrifugation, samples of gradient fractions were adjusted to a $1 \%$ concentration of sodium dodecyl sulfate and analyzed by agarose gel electrophoresis. The gel was incubated with RNase $(5 \mu \mathrm{g} / \mathrm{ml})$ at room temperature overnight to digest RNA, then stained with ethidium bromide. The gradient fraction numbers are at the top. Slot (M) has markers of MVMi single-stranded and RF DNAs. The position of band $\mathrm{X}$ in the $110 \mathrm{~S}$ peak is indicated. 


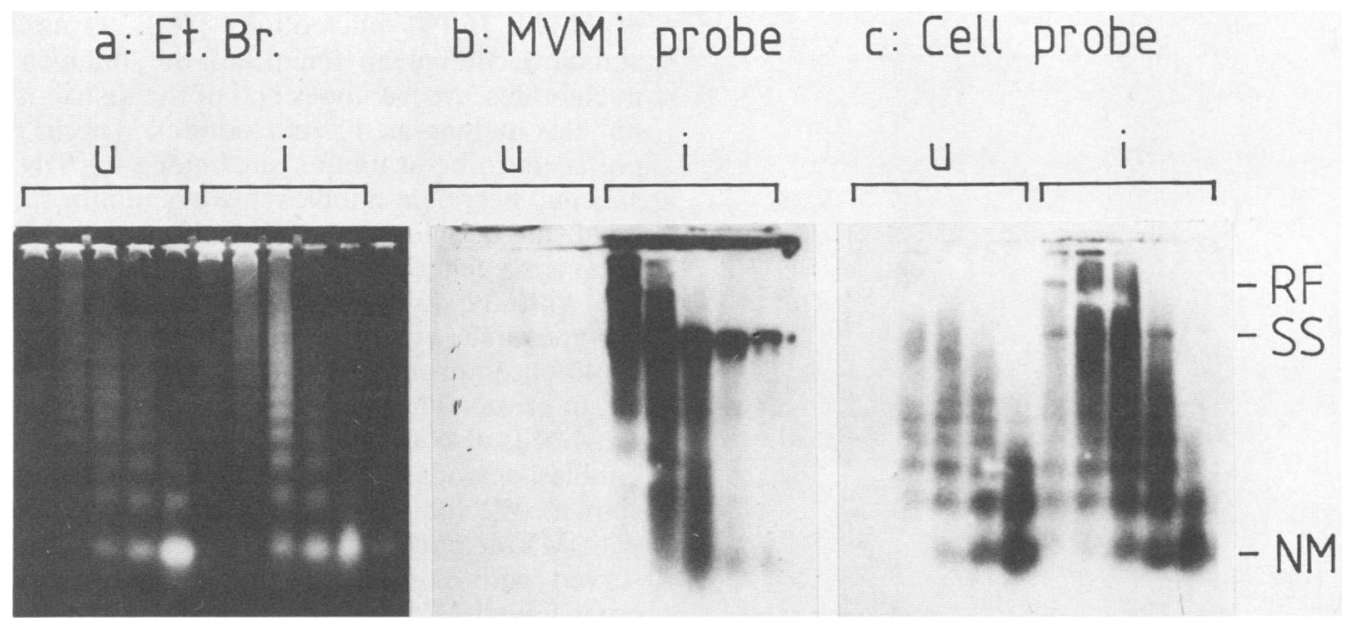

FIG. 6. Staphylococcal nuclease digestion of cellular chromatin and MVMi nucleoprotein complexes. Nuclei were isolated from MVMi-infected EL-4 cells and from uninfected cells. They were digested with staphylococcal nuclease for 0, 1, 2, 5, or 10 min, and the DNA fragments were isolated and analyzed by agarose gel electrophoresis as described in the Materials and Methods section. The DNA was stained with ethidium bromide (Et.Br.) (a), then transferred to nitrocellulose and hybridized with an MVMi-specific probe (b), and washed and rehybridized with a mouse-cell-specific probe (c). Lanes u, Samples for uninfected cells; lanes i, samples from MVMi-infected cells, with increasing times of digestion from left to right in each case. The positions of MVMi RF and single-stranded (SS) DNAs and that of the nucleosome monomer (NM) are shown.

length corresponding to a single nucleosome (compare with Fig. 6a and c).

To check that the transfer and hybridization method was capable of detecting nucleosomal DNA fragments, the same filter was washed under conditions that removed most of the MVMi DNA probe and rehybridized with radioactively labeled mouse DNA. The resulting autoradiogram (Fig. 6c) showed a nucleosomal repeat pattern for both uninfected

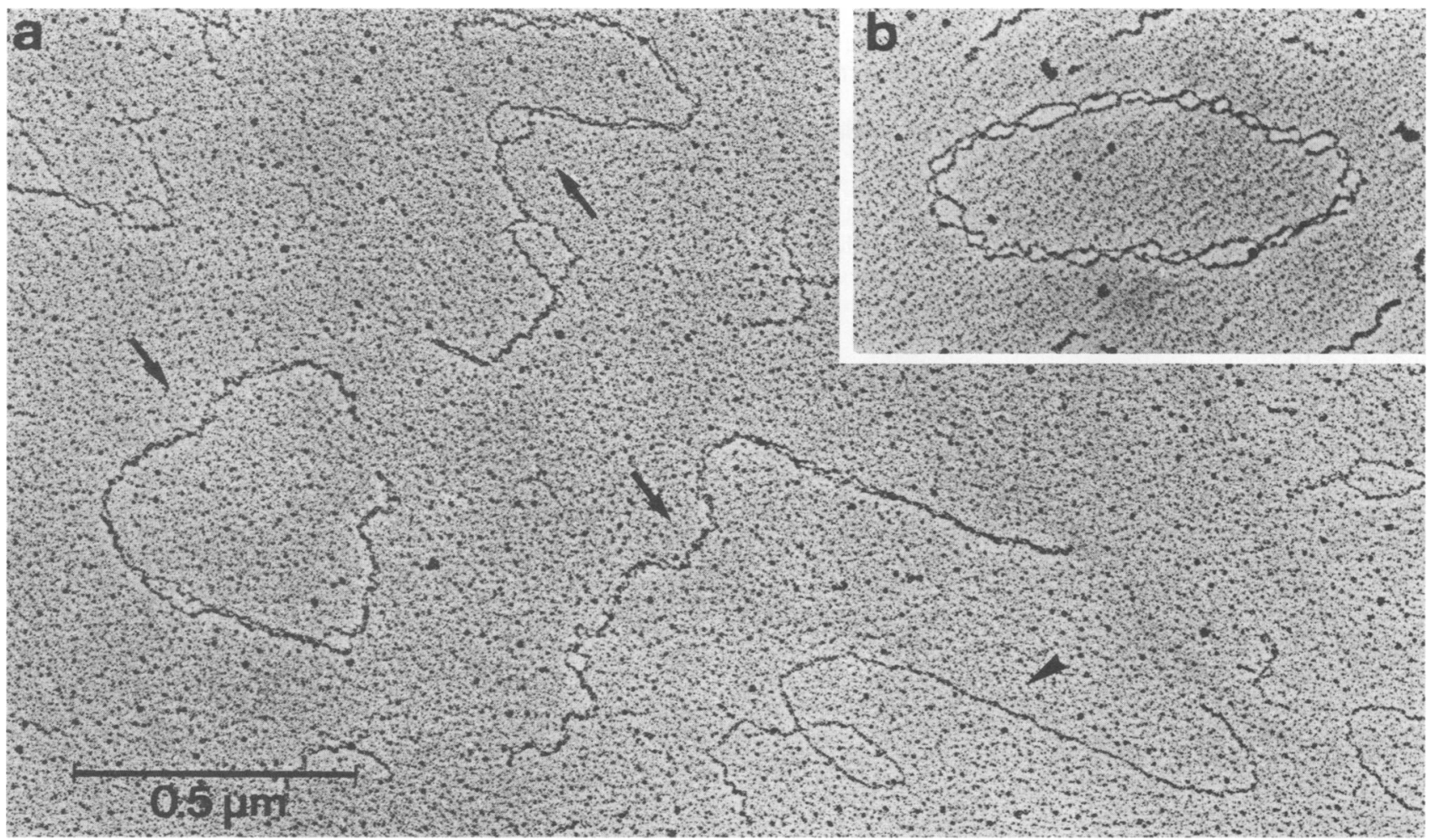

FIG. 7. Electron micrographs of denatured MVMi and SV40 DNA after psoralen cross-linking of MVMi nucleoprotein complexes and SV40 chromosomes. (a) MVMi DNA with molecules either mostly double stranded (shown by arrows) with single-stranded bubbles or wholly single stranded (shown by the arrow head). (b) SV40 DNA. 


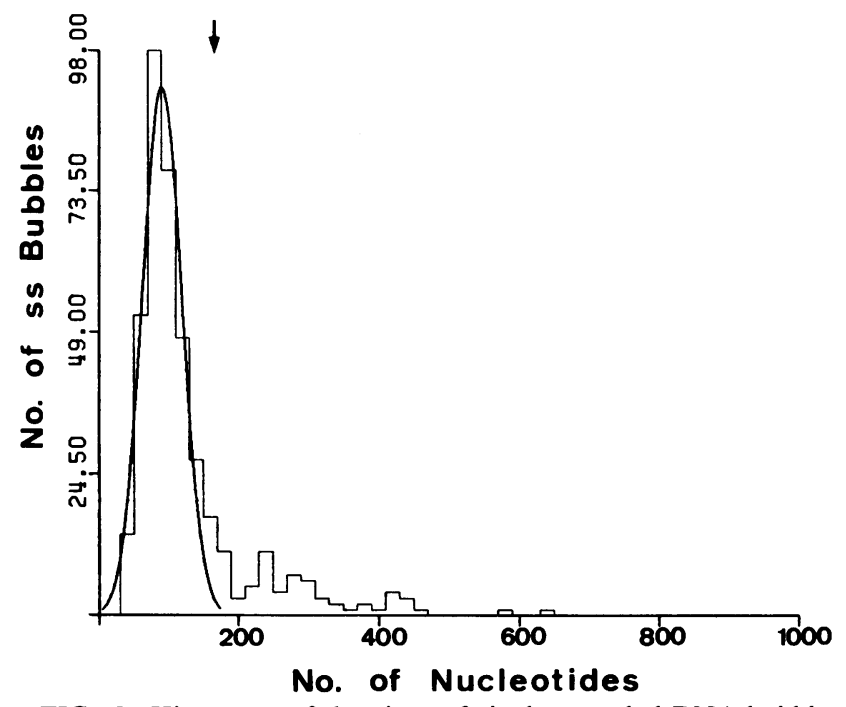

FIG. 8. Histogram of the sizes of single-stranded DNA bubbles in cross-linked MVMi RF DNA. DNA contour lengths were measured with a Hewlett-Packard digitizer (Hewlett-Packard Co., Palo Alto, Calif.) on photographic prints. The data were plotted by computer, using a class size of 20 nucleotides, and were fitted with a Gaussian distribution as described by Koller et al. (16). The length standard was the single-stranded MVMi DNA present in the preparation. For comparison, the mean size of the bubbles in the SV40 DNA (160 nucleotides; Sogo et al., J. Mol. Biol., in press) is indicated by an arrow.

and infected nuclei, with DNA accumulating in the nucleosomal monomer band on increasing nuclease digestion. Removal of the previously hybridized MVMi DNA apparently was incomplete, since the bands corresponding to the MVMi single-stranded and replicative form DNAs were still visible, though much fainter, in Fig. 6c.

Psoralen cross-linking of the DNA in MVMi nucleoprotein complexes. When double-helical DNA is irradiated with UV light in the presence of trimethylpsoralen, the DNA strands become cross-linked. The frequency of cross-links, under the conditions we used, is such that the DNA still appears uniformly double-stranded when prepared for electron microscopy under denaturing conditions (27). The presence of nucleosomes on the DNA interferes with either psoralen binding or the cross-linking reaction, so that the pattern of cross-linking of DNA in chromatin reflects the distribution of nucleosomes along the DNA, with the cross-links occurring in the internucleosomal spaces (27 and references therein).

We extracted MVMi nucleoprotein complexes as shown in Fig. 1, cross-linked the DNA with 4,5',8-trimethylpsoralen, and examined the distribution of cross-links in the purified DNA by electron microscopy under denaturing conditions. The cellular chromatin from the same preparations and extracted SV40 chromosomes (29) were treated in parallel as controls.

In the sample from MVMi infected cells (Fig. 7a), both single- and double-stranded DNAs were seen, representing, we assume, the virion form and RF DNAs known to be present in the extract. Denatured loops (bubbles) were present in the double-stranded DNA, confirming that there were indeed proteins on the DNA in the extracted MVMi nucleoprotein complexes. The sizes of the bubbles (Fig. 8) followed a narrow distribution somewhat skewed in the direction of longer bubbles. Using the single-stranded DNA of MVMi (5,081 nucleotides [26]), as an internal length standard, the mean length of the bubbles was $90 \pm 29$ nucleotides. At the upper end of the distribution, there were bubbles as long as 400 nucleotides. The larger bubbles did not seem to be at unique sites along the DNA, nor was the spacing between bubbles always uniform. On the other hand, the bubbles in the SV40 control sample (Fig. 7b) had an average length of $160 \pm 35$ nucleotides, which is consistent with the known nucleosomal structure of the SV40 chromosome (a detailed analysis of psoralen cross-linking of SV40 chromosomes is being published separately: Sogo et al., in press). The cross-linked chromosomal DNA from the EL-4 cells also showed the regularly spaced single-stranded bubbles characteristic of chromatin, as expected (data not shown). We conclude that the cross-linking pattern observed with MVMi nucleoprotein complexes differs from that observed with DNA from chromatin. In some samples of cross-linked MVMi DNA, double-stranded molecules held in a circular structure were observed. These may correspond to the circular structures noted by Bratosin et al. (7); we have not yet investigated them further.

\section{DISCUSSION}

We detected two forms of MVMi nucleoprotein complex in extracts of nuclei of infected cells. Neither of them apparently had a structure like that of the bulk of cellular chromatin, as judged by several criteria. The sedimentation coefficient of the faster-sedimenting complex (about 110S) is that of MVM virions (35). It contained single-stranded MVMi DNA and the viral capsid proteins. The singlestranded DNA was fairly resistant to staphylococcal nuclease. We conclude that the $110 \mathrm{~S}$ complex contained mature and possible immature MVMi virions. Another form of MVMi DNA (band X) was also detected in complexes sedimenting at $110 \mathrm{~S}$. This DNA was not present in purified mature MVMi virions, it migrated on electrophoresis between the single-stranded virion DNA and RF DNA, and after heating it migrated as the virion DNA. Band X DNA is unlikely to be double-stranded RF DNA containing a replication fork because this structure, being larger, is expected to migrate more slowly than RF on electrophoresis. Band X DNA could represent virion DNA held by base-pairing in a secondary structure (which could be associated with immature virus particles) or possibly a dimer of virion DNA held together by intermolecular base-pairing at the palindromic DNA ends. Nevertheless, the $110 \mathrm{~S}$ nucleoprotein complexes (in contrast to those sedimenting at 40S) did not incorporate labeled deoxynucleoside triphosphate into DNA in vitro.

The nucleoprotein complexes with a sedimentation coefficient of $40 \mathrm{~S}$ contained the double-stranded MVMi RF DNA. That this DNA was indeed associated with proteins is indicated by the following: the sedimentation coefficient was greater than that of purified MVMi RF DNA, which is 15S; the DNA was partially protected against cross-linking by psoralen; and when the $40 \mathrm{~S}$ material from a sucrose gradient was rebanded in an isopycnic gradient of metrizamide, it had the density of a nucleoprotein complex, not of bare DNA (data not shown). The relatively narrow size distribution of the DNA bubbles protected against psoralen suggests a repeated subunit structure.

The MVMi nucleoprotein complexes were sometimes contaminated with fragments of cellular chromatin. This was not simply a result of the chromatin extraction methods, since fragmented host cellular DNA was also seen when MVMi DNA was extracted directly from infected cells with 
sodium dodecyl sulfate (data not shown). Evidence of fragmentation of cellular chromatin can be seen in Fig. $6 \mathrm{c}$ in the first track (the zero time point for staphylococcal nuclease) from infected cells, which already shows a nucleosomal DNA repeat pattern. This is not seen in the sample from the uninfected control cells. Thus infection by MVMi leads to fragmentation of cellular chromatin. When a nuclear extract containing both the MVMi nucleoprotein complexes and fragments of cellular chromatin was analyzed by sucrose gradient velocity centrifugation and agarose gel electrophoresis, neither of the viral complexes sedimented at the same rate as did cellular chromatin with DNA of the length of MVMi DNA (Fig. 5).

To examine the proteins associated with MVMi DNA, we selected an extract which was not detectably contaminated with fragments of celluar chromatin, sedimenting in the same region of the gradient as the $\mathrm{MVMi}$ nucleoprotein complexes. Under these conditions, we did not detect histones cosedimenting with the viral complexes. In fact, the major proteins in the peak of nucleoprotein sedimenting at $110 \mathrm{~S}$ were the viral capsid proteins. It is still quite possible that each MVMi RF DNA molecule associated with a small amount of histone (not enough for us to detect) to form an irregular or unfolded structure. It is also possible that a small proportion of the intracellular MVMi DNA associated with histones to form regular chromatin, but the majority, apparently, did not. MVM virions are known not to contain histones (32). Identification of the proteins specifically associated with MVMi RF DNA proved difficult because of the number of host cell proteins which sedimented to the same position in sucrose gradients. Nevertheless we could readily detect the capsid proteins VP-1 and VP-2 in the 40 S peak. The cosedimentation does not prove that the capsid proteins are associated with the replicating complexes, but it would be at least consistent with the notion (33) that these proteins associate with newly synthesized progeny DNA strands. The MVM-coded nonstructural proteins, NS-1 and NS-2 (10), may well bind to intracellular viral DNA; this possibility can be studied when antibodies to the proteins are available.

When digested with staphylococcal nuclease, the intranuclear MVMi RF DNA, detected specifically by hybridization with an MVMi DNA probe, was degraded but did not form a nucleosomal DNA repeat pattern, nor did it accumulate quantitatively as the relatively nuclease-resistant nucleosome monomer. When, as an internal control, the DNA on the same filter was subsequently hybridized with a cellular DNA probe, the nucleosomal pattern was evident, showing that the methods used did not destroy nucleosomes. The intranuclear MVMi single-stranded DNA, in contrast, was degraded only very slowly by staphylococcal nuclease, even though this enzyme digests purified single-stranded DNA more rapidly than double-stranded DNA. We interpret this result as indicating that most of the intranuclear singlestranded DNA was within viral particles.

Ben-Asher et al. (4) have studied the intracellular organization of MVMp DNA in A9 cells. Like us, they detected two types of nucleoprotein complex, one sedimenting at $110 \mathrm{~S}$ and the other sedimenting at 50S. On the basis of electron microscopy and the observation of histones in the 110 S peak, they proposed that the $110 \mathrm{~S}$ peak contained both MVM minichromosomes and virions. The $50 \mathrm{~S}$ peak was suggested to comprise defective particles containing short DNA fragments. Our results do not support this interpretation. We observed a clear peak of MVMi RF DNA in the fraction sedimenting at $40 \mathrm{~S}$. Moreover, the $40 \mathrm{~S}$ nucleopro- tein complexes were able to incorporate labeled deoxynucleoside triphosphates into RF-sized DNA molecules in vitro, indicating that MVMi replicating intermediates sedimented at this position. It is not clear why our observations differ from those of Ben-Asher et al. (4). Although different cell and virus strains were used for most of the experiments, we found the sedimentation properties and staphylococcal nuclease digestion pattern of nucleoprotein complexes from MVMp-infected A9 cells to be similar to those we describe here for MVMi. An important difference may be in the methods used for extraction of the nucleoprotein complexes. Ben-Asher et al. (4) extracted the complexes at $37^{\circ} \mathrm{C}$ in the presence of $\mathrm{Mg}^{2+}$. These conditions favor the action of deoxyribonucleases, which could explain both their observation that the DNA in the more slowly sedimenting complex behaved as short pieces in an alkaline sucrose gradient and the observation of histones, presumably from fragmented cellular chromatin, in the sucrose gradient fractions containing the $110 \mathrm{~S}$ complex. In fact, the kinetics of labeling of the two complexes (4) (the $\left[{ }^{3} \mathrm{H}\right]$ thymidine was incorporated first into the more slowly sedimenting complex and only later appeared in the $110 \mathrm{~S}$ complex) support our view that the 40S complex contains the replicating DNA.

Why does intracellular MVMi DNA not form a regular chromatin structure? Since the viral DNA replicates in cells in the $S$ phase $(31$; reviewed in reference 5$)$, histones are presumably being synthesized. Two explanations, not mutually exclusive, occur to us. First, pulse-chase experiments (34) show that MVM RF DNA is an active intermediate in the viral lytic cycle, generating single-stranded progeny DNA. In addition, since the MVM RF DNA is presumably the template for transcription (3), a major portion of the intracellular MVMi RF DNA may be involved in the processes of transcription and replication. There is evidence to suggest that actively transcribed genes are not in a normal chromatin structure. For example, the nucleosomal repeat pattern of the Drosophila hsp70 gene disappears when transcription of the gene is induced (36). At the same time, the coding region of the gene becomes depleted of histones (15). MVMi RF DNA could represent actively transcribed DNA of this type.

Second, the MVMi RF is, at least for the most part, a short linear DNA molecule. The topological state of DNA may play a role in regulating chomatin structure. When linear or circular SV40 DNA is incubated in nuclei of Xenopus oocytes, the circular DNA is reconstituted into chromatin containing regularly spaced nucleosomes, whereas the linear DNA is not (23). Our observation that MVMi RF DNA did not form a regular chromatin structure may also indicate that in the infected mouse cells, chromatin can be assembled properly only on DNA that is able to be supercoiled. Whether this topological requirement for chromatin assembly is general, however, remains unclear. The intranuclear DNA of the parvovirus adeno-associated virus (AAV) was reported not to give a nucleosomal repeat pattern after staphylococcal nuclease digestion (8). However, in another study (21), partial nuclease digestion did reveal protected DNA fragments similar to those of nucleosomes. The fact that AAV DNA integrates into the host chromosomes (9) complicates interpretation of the results with AAV. The linear genome of adenovirus itself (11) and that of herpes simplex virus (19) are found predominantly in structures other than nucleosomes during the late phase of viral replication. A fraction of the parental adenovirus DNA on the other hand, in the early phase of infection, apparently does form a nucleosome-like structure (30). 


\section{ACKNOWLEDGMENTS}

We thank B. Bentele for help with tissue culture, A.-M. Vodoz for typing the manuscript, and V. Jongeneel and B. Hirt for many helpful discussions.

This work was supported by grants 3.261 .82 and 3.585 .84 from the Fonds National Suisse de la Recherche Scientifique.

\section{LITERATURE CITED}

1. Astell, C. R., M. Thomson, M. Merchlinsky, and D. C. Ward. 1983. The complete DNA sequence of minute virus of mice, an autonomous parvovirus. Nucleic Acids Res. 11:999-1018.

2. Beard, P., and K. Nyfeler. 1982. Transcription of simian virus 40 chromosomes in an extract of HeLa cells. EMBO J. 1:9-14.

3. Ben-Asher, E., and Y. Aloni. 1984. Transcription of minute virus of mice, an autonomous parvovirus, may be regulated by attenuation. J. Virol. 52:266-276.

4. Ben-Asher, E., S. Bratosin, and Y. Aloni. 1982. Intracellular DNA of the parvovirus minute virus of mice is organized in a minichromosome structure. J. Virol. 41:1044-1054.

5. Berns, K. (ed.). 1984. The parvoviruses. Plenum Publishing Corp., New York.

6. Bonnard, G. D., E. K. Manders, D. A. Campbell, R. B. Herberman, and M. J. Collins. 1976. Immunosuppressive activity of a subline of the mouse EL-4 lymphoma. J. Exp. Med. 143: 187-205.

7. Bratosin, S., O. Laub, Y. Tal, and Y. Aloni. 1979. Mechanism of circularization of linear DNAs: circular parvovirus MVM DNA is formed by a noose sliding in a lasso-like DNA structure. Proc. Natl. Acad. Sci. USA 76:4289-4293.

8. Brown, M., and J. Weber. 1982. Adeno-associated virus has a unique chromatin structure. Can. J. Biochem. 60:1001-1005.

9. Cheung, A. K. M., M. D. Hoggan, W. W. Hauswirth, and K. I. Berns. 1980. Integration of the adeno-associated virus genome into cellular DNA in latently infected human Detroit 6 cells. J. Virol. 33:739-748.

10. Cotmore, S. F., L. J. Sturzenbecker, and P. Tattersall. 1983. The autonomous parvovirus MVM encodes two nonstructural proteins in addition to its capsid polypeptides. Virology 129: 333-343.

11. Daniell, E., D. E. Groff, and M. J. Fedor. 1981. Adenovirus chromatin structure at different stages of infection. Mol. Cell Biol. 1:1094-1105.

12. Engers, H. D., J. A. Louis, R. H. Zubler, and B. Hirt. 1981. Inhibition of T cell-mediated functions by MVM(i), a parvovirus closely related to minute virus of mice. J. Immunol. 127: 2280-2285.

13. Felsenfeld, G. 1978. Chromatin. Nature (London) 271:115-122.

14. Hübscher, U. 1983. The mammalian primase is part of a high molecular weight DNA polymerase polypeptide. EMBO J. 2:133-136.

15. Karpov, V. L., O. V. Preobrazhanskaya, and A. D. Mirzabekov. 1984. Chromatin structure of hps70 genes, activated by heat shock: selective removal of histones from the coding region and their absence from the $5^{\prime}$ region. Cell 36:423-431.

16. Koller, T., O. Kübler, R. Portmann, and J. M. Sogo. 1978. High resolution physical mapping of specific binding sites of $\mathrm{E}$. coli RNA polymerase on the DNA on bacteriophage T7. J. Mol. Biol. 120:121-131.

17. Laemmli, U. K. 1977. Cleavage of structural proteins during the assembly of the head of bacteriophage T4. Nature (London) 227:680-685.
18. Laskey, R. 1980. The use of intensifying screens or organic scintillators for visualizing radioactive molecules resolved by gel electrophoresis. Methods Enzymol. 65:363-371.

19. Leinbach, S. S., and W. C. Summers. 1980. The structure of herpes simplex virus type I DNA as probed by micrococcal nuclease digestion. J. Gen. Virol. 51:49-59.

20. Maniatis, T., E. F. Fritsch, and J. Sambrook. 1982. Molecular cloning: a laboratory manual, p. 387-389. Cold Spring Harbor Laboratory, Cold Spring Harbor, N.Y.

21. Marcus-Sekura, C. J., and B. J. Carter. 1982. Chromatin-like structure of adeno-associated virus DNA in infected cells. J. Virol. 48:79-87.

22. McMaster, G. K., P. Beard, H. D. Engers, and B. Hirt. 1981. Characterization of an immunosuppressive parvovirus related to the minute virus of mice. J. Virol. 38:317-326.

23. Mertz, J. E. 1982. Linear DNA does not form chromatin containing regularly spaced nucleosomes. Mol. Cell. Biol. 2:1608-1618

24. Oakley, B. R., D. R. Kirsch, and N. R. Morris. 1980. A simplified ultrasensitive silver strain for detecting proteins in polyacrylamide gels. Anal. Biochem. 105:361-363.

25. Rigby, P. W. J., M. Dieckmann, C. Rhodes, and P. Berg. 1977. Labeling deoxyribonucleic acid to high specific activity in vitro by nick translation with DNA polymerase I. J. Mol. Biol. 113:237-251.

26. Sahli, R., G. K. McMaster, and B. Hirt. 1985. DNA sequence comparison between two tissue-specific variants of the autonomous parvovirus, minute virus of mice. Nucleic Acids Res. 13:3617-3633.

27. Sogo, J. M., P. J. Ness, R. M. Widmer, R. W. Parish, and T. Koller. 1984. Psoralen-crosslinking of DNA as a probe for the structure of active nucleolar chromatin. J. Mol. Biol. 178: 897-928.

28. Southern, E. M. 1975. Detection of specific sequences among DNA fragments separated by gel electrophoresis. J. Mol. Biol. 98:503-517.

29. Su, R. T., and M. L. DePamphilis. 1978. Simian virus 40 DNA replication in isolated replicating viral chromosomes. J. Virol. 28:53-65.

30. Tate, V. E., and L. Philipson. 1979. Parental adenovirus DNA accumulates in nucleosome-like structures in infected cells. Nucleic Acids Res. 6:2769-2785.

31. Tattersall, P. 1972. Replication of the parvovirus MVM. I. Dependence of virus multiplication and plaque formation on cell growth. J. Virol. 10:586-590.

32. Tattersall, P., P. J. Cawte, A. J. Shatkin, and D. C. Ward. 1976. Three structural polypeptides coded for by minute virus of mice, a parvovirus. J. Virol. 20:273-289.

33. Tattersall, P., and D. C. Ward. 1976. The rolling hairpin: a model for the replication of parvovirus and linear chromosomal DNA. Nature (London) 263:106-109.

34. Ward, D. C., and D. K. Dadachanji. 1978. Replication of minute-virus-of-mice DNA, p. 297-313. In D. C. Ward and P. Tattersall (ed.), Replication of mammalian parvoviruses. Cold Spring Harbor Laboratory, Cold Spring Harbor, N.Y.

35. Ward, D. C., and P. Tattersall (ed.). 1978. Replication of mammalian parvoviruses. Cold Spring Harbor Laboratory, Cold Spring Harbor, N.Y.

36. Wu, C., Y. G. Wong, and S. C. R. Elgin. 1979. The chromatin structure of specific genes. II. Disruption of chromatin structure during gene activity. Cell 16:807-814. 